boundaries at just about the scale where one might have expected to see a real change in the slope of the correlation function. Thus only effects that have been seen in more than one survey should be taken seriously, such as the slope of the correlation function $\left(r^{-1.8}\right)$, but not its precise amplitude, the length scale at which it turns over (if it does), or whether it goes negative at some large distance.

Clusters of galaxies are, themselves, even more strongly clustered than the galaxies, according to Neta Bahcall (STScI). Rich clusters are more clumped than poorer ones. That is, the amplitude of the richcluster correlation function exceeds that of the galaxy correlation function by a factor of about 30 , at a scale length of $5 \mathrm{Mpc}$; the amplitude for smaller clusters falls in between; and the slope of all three correlation functions is $r^{-1.8}$. Bahcall interprets this to mean that a large fraction of galaxies form outside clusters. But Schramm pointed out that, when the correlation functions are renormalized to scale length as a fraction of the average separation of the objects concerned (a technique adopted from condensed-matter physics), rich and poor clusters look the same, while the galaxies are more strongly clustered, reflecting the action of an extra factor, presumably gravity. If this is correct, then the galaxy correlation function should go negative at about $40 \mathrm{Mpc}$ and that of the cluster at 400 Mpc. As Geller noted, some surveys show such a turn-over but others do not.

Finally, Bahcall remarked that clustering on still larger scales should reveal itself through large velocity deviations from smooth expansion. A hint of the predicted peculiar velocities has recently turned up at another meeting ${ }^{\dagger}$. Marc Aaronson (University of Arizona) and Jeremy Mould (California Institute of Technology) have analysed redshift data for 10 nearby clusters, the distances of which have been determined from the correlation of infrared luminosity with $21 \mathrm{~cm}$ line width (the TullyFisher relation). Scatter in the derived value of the Hubble constant is minimized if the Local Group of galaxies has a velocity of $788 \pm 188 \mathrm{~km} / \mathrm{s}$ in the direction of galactic latitude $18^{\circ} \pm 13^{\circ}$ and longitude $255^{\circ} \pm$ $17^{\circ}$. This is in reasonable agreement with the $600 \pm 30 \mathrm{~km} / \mathrm{s}$ in about the same direction that seems to be our velocity relative to the $3 \mathrm{~K}$ microwave radiation. But, more to the point, it can be broken up into a vector sum of an infall of the Local Group toward the centre of our (Virgo) supercluster at about $300 \mathrm{~km} / \mathrm{s}$ plus a somewhat larger motion of the supercluster towards the adjacent Hyades-Centaurus supercluster. The best-fit value of the Hubble contant from this analysis is about 90 $\mathrm{km} / \mathrm{s} / \mathrm{Mpc}$, which presents its own problems. But that's another story.

Virginia Trimble is at the University of California, Irvine, California 92717 and the University of Maryland, Maryland 20742, USA.

TThe 165 th meeting of the American Astronomical Society, 14-16 January 1985, Tucson, Arizona.

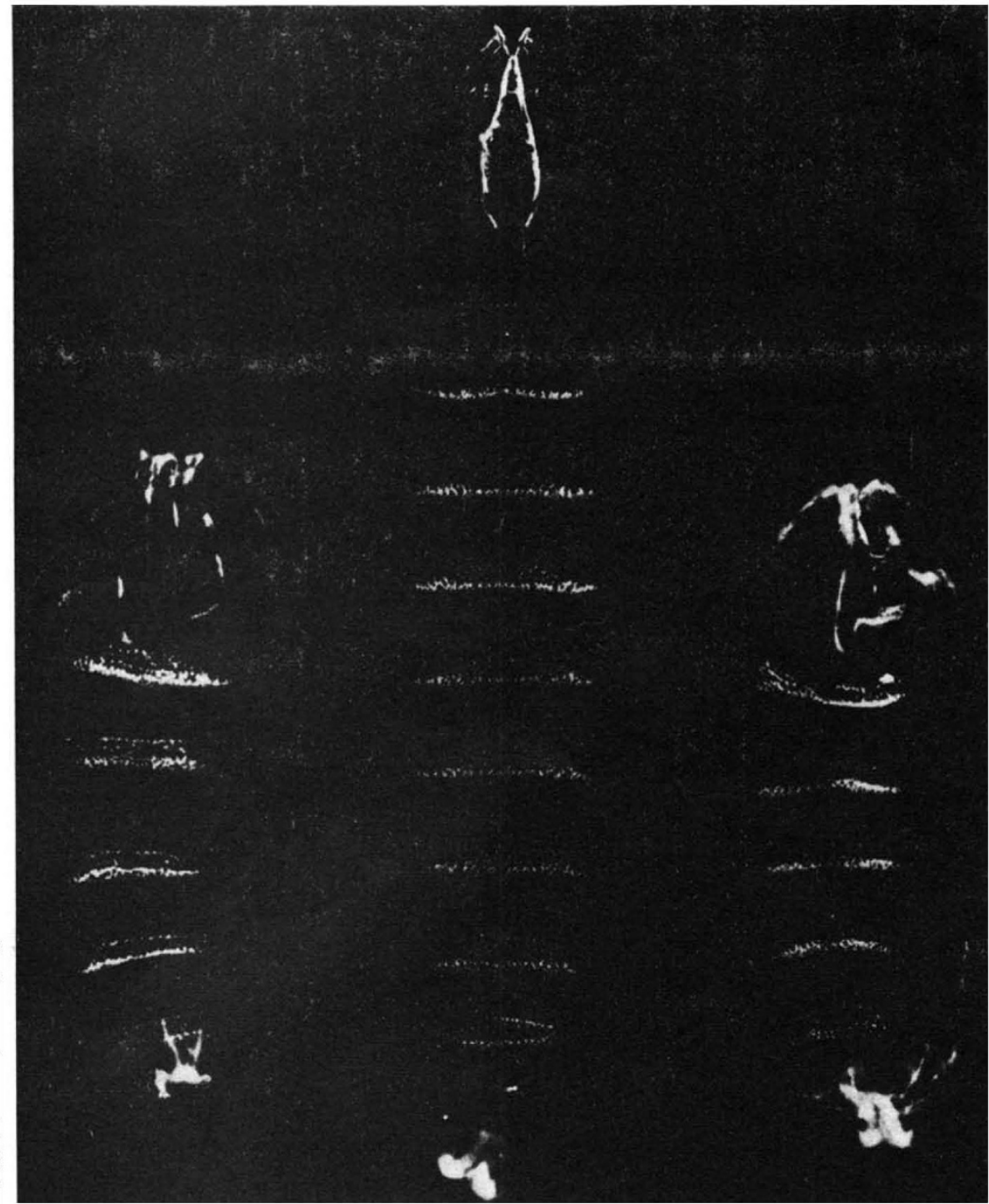

\section{Mutants without mutations}

THE Drosophila embryo shown on the left is an example of a Krüppel mutant, the result of a mutation in the Krüppel gene. This embryo is short relative to the normal embryo shown in the middle, because it lacks all thoracic and several abdominal segments, so that the head is directly connected to one of the more posterior abdominal segments. The embryo on the right, which resembles the Krüppel mutant, in fact has normal Krüppel genes, but is the dramatic result of the injection of the embryo, at a very early stage in its development, with a large dose of what is known as Krüppel 'antisense' RNA, as described on page 703 of this issue.

Krüppel 'antisense' RNA, which is synthesized in vitro from the 'wrong' strand of Krüppel cDNA, is complementary in sequence to Krüppel 'sense' (natural) RNA and so is able to pair with it to form doublestranded RNA. Although the precise fate of Krüppel antisense RNA in injected embryos is unknown, it is thought to pair with Krüppel sense RNA and so prevent it from being translated into the normal Krüppel gene product. Direct evidence that antisense RNA (for beta globin) works in this way in microinjected frog oocytes has recently been reported by D.A. Melton (Proc. natn. Acad. Sci. 82, 144; 1985).

The ability to 'phenocopy' mutants in this way is of more than academic interest. An example of the kind of problem to which the technique might be applied comes from some recent work on the engrailed locus of Drosophila, which was discussed by Peter Lawrence in these pages three weeks ago (24 January, p.268). Engrailed has some characteristics of a homoeotic gene, and, like the better known homoeotic genes of the bithorax and Antennapedia complexes, it contains a homoeo-box sequence. Close by the engrailed gene there is a second homoeobox sequence, which identifies the engrailed-related gene. What does this gene do? Nobody knows, for as yet no mutations mapping in this region of the Drosophila genome have been identified. The use of antisense RNA offers a direct way to test what effect the loss of engrailed-related gene function has on development. Antisense RNA should also be useful in dissecting the activities of genes involved in the regulation of development by preventing their expression at specific times and in discrete regions of the embryo.

Geoffrey North 